\title{
A NON-PATHOGENIC MYCOPLASMA INHIBITING THE EFFECT OF A PATHOGENIC MYCOPLASMA IN ORGAN CULTURE
}

\author{
D. TAYlor-Robinson AND J. D. Cherry* \\ M.R.C. Clinical Research Centre, Watford Road, Harrow, Middlesex
}

MiXeD infections of mycoplasmas and bacteria, mycoplasmas and viruses, and all three agents have been studied in animals, and particularly in birds (Blake, 1962; Fabricant, 1969). The enhancement or inhibition by mycoplasmas of the growth of viruses and their cytopathic effects in tissue cultures has been studied by numerous workers (Macpherson, 1966; Hargreaves and Leach, 1970). Recently, various authors have described the growth and damaging effects of mycoplasmas in organ cultures, particularly organ cultures of respiratory epithelium (Butler, 1969; Collier, Clyde and Denny, 1969; Cherry and Taylor-Robinson, 1970a). Reed (1971) studied the effect of a mixed infection of Mycoplasma hyorhinis and swine influenza virus in pig tracheal organ cultures and observed that the virus enhanced the growth of the mycoplasma. We found that various strains of $M$. gallisepticum stopped the ciliary activity of chicken tracheal organ cultures (Cherry and Taylor-Robinson, $1970 a, 1971)$ and we reasoned that the presence of another mycoplasma, namely $M$. gallinarum, might enhance this effect. The present report shows, however, that the second mycoplasma actually protected the ciliated epithelium against the inhibitory effect of $M$. gallisepticum.

\section{MATERIALS AND METHODS}

Organ cultures. The method of preparation has been described in detail elsewhere (Cherry and Taylor-Robinson, $1970 a$ ). Briefly, rings, $0 \cdot 5-1 \mathrm{~mm}$ thick, were cut from the tracheas of 19-20-day-old chicken embryos. Each ring was placed in a screw-capped tissue-culture tube containing $1 \mathrm{ml}$ of medium and incubated in a roller drum at $37^{\circ} \mathrm{C}$. The rings adhered to the glass during rolling; they were detached every 24 to $48 \mathrm{hr}$ by shaking the tubes and then allowed to re-adhere.

Media. The medium for organ cultures was Eagle's basal medium with 200 units of penicillin $\mathrm{G}$ per $\mathrm{ml}$ and $0.05 \mathrm{M} \mathrm{N}$-hydroxy-ethylpiperazine- $\mathrm{N}^{\prime}$-2-ethanesulphonic acid (HEPES) buffer; the medium was not supplemented with serum. The $p \mathrm{H}$ was adjusted to 7.0 by the addition of $0.2 \mathrm{~N}-\mathrm{NaOH}$.

The medium for the growth and titration of mycoplasmas has been described previously (Manchee and Taylor-Robinson, 1968). Thallium acetate was omitted from the medium used to grow mycoplasmas for inoculation into organ culture.

Mycoplasmas. The origins of the various strains of M. gallisepticum (table II) are given elsewhere (Taylor-Robinson and Berry, 1969), as are the sources of M. mycoides var. capri and $M$. hominis (Cherry and Taylor-Robinson, 1970a). The strains of M. gallinarum were

Received 30 Sept. 1971; accepted 10 Nov. 1971.

* Visiting worker from the Department of Pediatrics, St Louis University School of Medicine, St Louis, Missouri.

J. MED. MICROBIOL.-VOL. 5 (1972)

291

U 
obtained from Dr D. H. Roberts (Central Veterinary Laboratory, Weybridge). All strains were cloned on agar medium and passed once in liquid medium without thallium acetate. Portions of this were stored at $-70^{\circ} \mathrm{C}$.

Titration of mycoplasmas. Serial ten-fold dilutions were made in mycoplasma liquid medium containing phenol red. For titrations of $M$. gallisepticum the medium was adjusted to $p \mathrm{H} 7.8$ and contained 0.1 per cent. glucose; for $M$. gallinarum the $p \mathrm{H}$ was 7.0 and the medium contained $0 \cdot 1$ per cent. arginine. The greatest dilution at which a colour change occurred during incubation at $37^{\circ} \mathrm{C}$ for 7 days was taken as the end-point of the titration; it was considered to contain one colour-changing unit (CCU). In mixed infections, where a

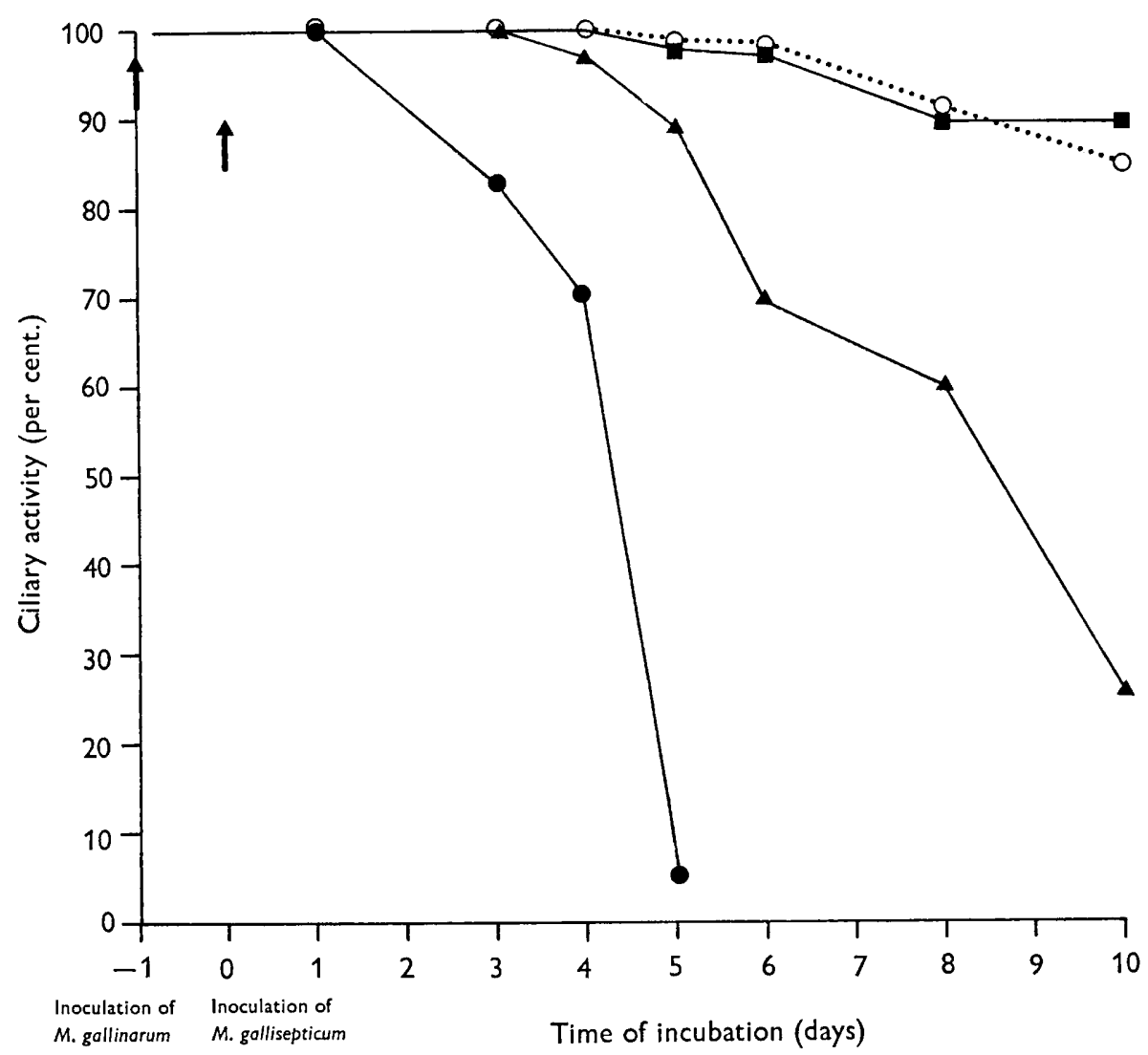

FIG. 1.-The reduction of ciliary activity of chicken tracheal organ cultures caused by $M$. gallisepticum (strain J1), alone and in the presence of M. gallinarum (introduced $24 \mathrm{hr}$ previously). $\bigcirc-0-0$ control, uninfected cultures; -1 M. gallisepticum alone;

cum + M. gallinarum; M. gallinarum alone.

more accurate assessment of the numbers of organisms was needed, titrations were done on solid medium. Colonies of $M$. gallinarum develop more quickly than those of $M$. gallisepticum so that the two organisms can be easily differentiated in mixed infections. In the case of specimens containing both species, colony counts at 2 days represented M. gallinarum, and counts at 7 days the combined $M$. gallinarum- $M$. gallisepticum content; the $M$. gallisepticum count was calculated by subtracting the 2-day count from the 7-day count. Results were recorded as colony-forming units (CFU) per $0.1 \mathrm{ml}$.

M. gallisepticum colonies haemadsorb; M. gallinarum colonies do not (Manchee and Taylor-Robinson, 1968). However, attempts to recognise the colonies of the two 
mycoplasmas in mixed cultures by overlaying them with erythrocytes were only partially successful as colonies were often lost from the agar surface by this procedure.

Ciliary activity. The method of observing and grading ciliary activity has been described previously (Cherry and Taylor-Robinson, 1970a). Both the extent and the vigour of ciliary activity are assessed and the results are combined and recorded as percentage ciliary activity. A group of five cultures was used for the assessment of each factor.

TABLE I

The inhibition by M. gallinarum of the cilia-stopping effect of M. gallisepticum (J1 strain) in chicken tracheal organ cultures

\begin{tabular}{|c|c|c|c|}
\hline \multirow{2}{*}{$\begin{array}{l}\text { Experiment } \\
\text { number }\end{array}$} & \multicolumn{2}{|c|}{$\begin{array}{c}\text { Time (days) required for } 50 \text { per cent. } \\
\text { reduction in ciliary activity by } \\
M . \text { gallisepticum }\end{array}$} & \multirow{2}{*}{$\begin{array}{l}\text { Strain of } \\
M . \text { gallinarum } \\
\text { used }^{*}\end{array}$} \\
\hline & alone & $\begin{array}{l}\text { in the presence of } \\
M . \text { gallinarum }\end{array}$ & \\
\hline $\begin{array}{l}1 \\
2 \\
3 \\
4 \\
5 \\
6 \\
7\end{array}$ & $\begin{array}{l}4.4 \\
2.4 \\
5 \cdot 6 \\
4.5 \\
5.4 \\
5.6 \\
3.0\end{array}$ & $\begin{array}{r}8.6 \\
4.8 \\
>10.0 \\
17.0 \\
9 \cdot 2 \\
9 \cdot 6 \\
6.2\end{array}$ & $\begin{array}{c}\text { W115 } \\
\text { W115 } \\
\text { W115 } \\
\text { W115 } \\
\text { W115 } \\
\text { Weybridge } \\
\text { PG16 }\end{array}$ \\
\hline $\begin{array}{c}\text { Average of } \\
\text { seven experiments }\end{array}$ & $4 \cdot 4$ & $9 \cdot 3$ & \\
\hline
\end{tabular}

* Introduced into the cultures 24 to $48 \mathrm{hr}$ before $M$. gallisepticum.

\section{RESULTS}

Inhibition by M. gallinarum of the cilia-stopping effect (CSE) of M. gallisepticum

M. gallisepticum strain J1. From previous experiments (Cherry and TaylorRobinson, 1970a, 1971) it was known that the J1 strain of M. gallisepticum had a rapid CSE when introduced into chicken tracheal organ cultures, an inoculum of $10^{3} \mathrm{CCU}$ causing a 50 per cent. reduction in ciliary activity within 5 days. In seven experiments (table I) it was observed that a dose of $10^{3}$ to $10^{6} \mathrm{CCU}$ of $M$. gallinarum, added to the organ culture 24 to $48 \mathrm{hr}$ before the J1 strain, inhibited this effect; the median time elapsing before there was a 50 per cent. reduction in CSE was now doubled. A representative experiment is shown in fig. 1. Two other strains of M. gallinarum (Weybridge and PG 16) were also capable of preserving the ciliary activity of organ cultures infected with $M$. gallisepticum (table I).

Other strains of $M$. gallisepticum. The ability of $M$. gallinarum to inhibit the CSE of ten other strains of M. gallisepticum was tested. Table II shows that inhibition was obtained with six of the ten strains. When individual strains of M. gallisepticum were tested on more than one occasion the results were always the same. 
Time interval before adding $M$. gallinarum. The effect was examined of adding $M$. gallinarum to chicken tracheal organ cultures at the same time as the $\mathrm{J} 1 \mathrm{strain}$ of $M$. gallisepticum, or $24 \mathrm{hr}, 48 \mathrm{hr}$ or $96 \mathrm{hr}$ before the $\mathrm{J} 1$ strain. The CSE was most markedly inhibited when $M$. gallinarum preceded the J1 strain by 24 or $48 \mathrm{hr}$. It was not significantly diminished when the two mycoplasmas were inoculated concurrently.

The effect of killed $M$. gallinarum. Chicken tracheal organ cultures were seeded either with $10^{6} \mathrm{CCU}$ of $M$. gallinarum or with the same inoculum after it had been heated at $56^{\circ} \mathrm{C}$ for $20 \mathrm{~min}$. The $\mathrm{J} 1$ strain of $M$. gallisepticum was added $\left(10^{5} \mathrm{CCU}\right)$ to both sets of cultures $24 \mathrm{hr}$ later. The cultures containing

TABLE II

Susceptibility of the cilia-stopping effect (CSE) of various strains of M. gallisepticum to inhibition by $M$. gallinarum

\begin{tabular}{l|c|c}
\hline $\begin{array}{c}\text { Strain of } \\
\text { Mallisepticum } \\
\text { tested }\end{array}$ & $\begin{array}{c}\text { Inhibition of CSE } \\
\text { by M. gallinarum }\end{array}$ & $\begin{array}{c}\text { Number of tests } \\
\text { carried out }\end{array}$ \\
\hline J1 & + & 7 \\
A514 & + & 4 \\
C54 & + & 4 \\
T34 & + & 4 \\
X95 & + & 1 \\
MG1 & + & 1 \\
W57 & + & 1 \\
F565 & - & 2 \\
A5969 & - & 1 \\
T37 & - & 1 \\
\hline
\end{tabular}

viable $M$. gallinarum showed inhibition of CSE, as described previously, but those with dead $M$. gallinarum lost ciliary activity at the same rate as control organ cultures containing only $M$. gallisepticum.

Growth of M. gallinarum and M. gallisepticum in mixed infections. A possible explanation for the inhibitory phenomenon observed in the mixed infections was inhibition of the growth of $M$. gallisepticum by M. gallinarum. However, the results of three representative experiments (table III) show that the growth of $M$. gallisepticum was not reduced by the presence of M. gallinarum.

\section{Activity of other mycoplasmas}

It was already known that the pathogenic goat mycoplasma $M$. capri had a CSE in chicken tracheal organ cultures (Cherry and Taylor-Robinson, 1970b). In further tests, $M$. capri produced a 50 per cent. loss of ciliary activity in $4 \cdot 2$ days. When $M$. gallinarum was also added, $24 \mathrm{hr}$ before $M$. capri, this amount of loss of activity was seen only after 6.2 days. $M$. gallinarum therefore probably possesses some activity also against $M$. capri.

The possibility that mycoplasmas other than $M$. gallinarum were capable of inhibiting the CSE of $M$. gallisepticum was investigated. Cultures were seeded 
with $10^{7} \mathrm{CCU}$ of the human genital mycoplasma, $M$. hominis, $24 \mathrm{hr}$ before $M$. gallisepticum. The rate of loss of ciliary activity of the tracheal rings in these cultures was the same as in cultures infected with $M$. gallisepticum only.

\section{Effect of $M$. gallinarum alone on organ cultures}

In several of the preceding experiments, ciliary activity of the tracheal rings persisted for longer in cultures infected with $M$. gallinarum only than in un-

TABLE III

Growth of M. gallisepticum (strain JI) and M. gallinarum in chicken tracheal organ cultures separately and in mixed infections

\begin{tabular}{c|c|c|c|c|c}
\hline & & \multicolumn{3}{|c}{ Mycoplasma growth (colony-forming units per 0.1 ml of } \\
organ culture medium)
\end{tabular}

* Day 0 is when $M$. gallisepticum was added to the organ cultures. M. gallinarum was always inoculated before $M$. gallisepticum and this is recorded as a minus number of days.

$\dagger$ Concentration of organisms immediately after their inoculation into the cultures.

inoculated "control" cultures. This was demonstrated most convincingly (fig. 2) when efforts were made to preserve ciliary activity for as long as possible by changing the medium at 3- to 6-day intervals. In $M$. gallinarum-infected cultures, a chronic infection was established; the organisms were present ( $10^{4} \mathrm{CCU}$ per $\mathrm{ml}$ ) 41 days after the cultures were initiated, even though the medium had been changed eight times. At this time there was less than 50 per cent. loss of ciliary activity in these cultures, as compared with a 50 per cent. loss in uninoculated cultures after 21 days.

\section{Discussion}

M. gallisepticum is pathogenic for the respiratory tract of poultry (Fabricant, 1969). The distinct species, M. gallinarum (Edward and Freundt, 1956; Roberts, 1964), has not been shown to be a pathogen, although it is frequently found in the respiratory tract. In the organ-culture experiments there was no 
doubt that the damaging effects produced by most, but not all, strains of $M$. gallisepticum were inhibited by the presence of $M$. gallinarum. Furthermore, this protection of the ciliated epithelium was demonstrable with all the strains of $M$. gallinarum that were tested. The single strain of $M$. hominis examined did not appear to possess protective activity, but whether $M$. gallinarum is unique in this respect it is not possible to say at this stage. There was some evidence that the cilia-stopping effect of $M$. capri was also inhibited by $M$. gallinarum. The phenomenon may not, therefore, be confined to M. gallisepticum-M. gallinarum mixed infections.

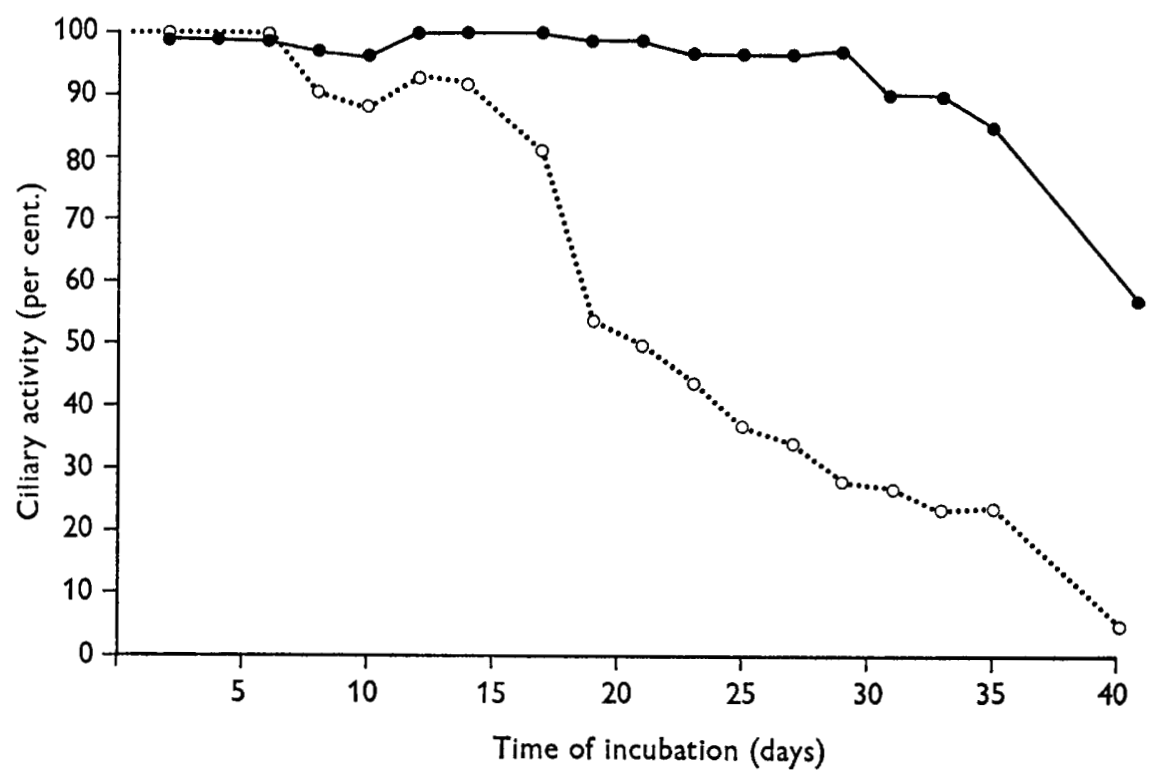

FIG. 2.-Prolongation of ciliary activity of chicken tracheal organ cultures infected with M. gallinarum. M. gallinarum; $\bigcirc-\ldots$ control, uninfected cultures.

The mechanism of protection is far from clear. To provide a marked protective effect $M$. gallinarum must be present in the organ cultures before the introduction of $M$. gallisepticum. The presence of $M$. gallinarum does not seem to inhibit the growth of $M$. gallisepticum, but the earlier inoculation of the former into the cultures may allow them to grow and form a barrier between the $M$. gallisepticum organisms and the ciliated epithelial cells; this might block the approach of toxins, which are likely to be liberated by $M$. gallisepticum (Thomas, 1970; Cherry and Taylor-Robinson, 1971). However, there is a more plausible explanation for the protective phenomenon. Rather surprisingly, control, uninoculated organ cultures were found to lose ciliary activity more rapidly than did cultures infected with $M$. gallinarum only. This was almost certainly not due to low-grade, egg-transmitted $M$. gallisepticum infection of the organ cultures. All attempts to detect such natural infection by cultural methods were unsuccessful, and the loss of ciliary activity in the control cultures was much slower than would be expected if they contained M. gallisepticum. 
It is known that chicken tracheal organ cultures produce peroxide (Cherry and Taylor-Robinson, 1970c) and this may be responsible, at least in part, for their eventual autolysis. Mycoplasmas themselves produce peroxide which accounts for their haemolytic activity, but at the same time several species also produce a substance, possibly a peroxidase, that protects erythrocytes from autolysis (Manchee and Taylor-Robinson, 1970). In the same way, M. gallinarum may produce a substance that destroys peroxide in the tracheal cultures; there is some support for this from previous studies (Cherry and Taylor-Robinson, $1970 c$ ) in which we found less peroxide in M. gallinarum-infected cultures than in uninoculated, control cultures. It is interesting that only viable M. gallinarum organisms possess protective activity, perhaps because only while actively metabolising can they produce peroxidase, or other soluble metabolic products, responsible for protecting cultures against degenerative autolytic processes and against the damaging effects of infection with $M$. gallisepticum.

Some strains of $M$. gallisepticum were unaffected in their cilia-stopping activity by the presence of $M$. gallinarum, and it is possible that, in natural infections of poultry, such strains are the more pathogenic for the respiratory epithelium. Numerous mycoplasma species have been recognised in poultry and the effect of one on another may have a bearing on the outcome of infection by them, and indeed on mycoplasma ecology. Of course, this concept need not be confined to avian species, and the interrelationships between mycoplasmas in other hosts, such as man, seem to be worth exploring.

\section{SUMMARY}

The pathogenic avian mycoplasma $M$. gallisepticum caused a 50 per cent. reduction of ciliary activity in chicken tracheal organ cultures after 4-5 days. Nine days were required for a similar loss of activity when the non-pathogenic mycoplasma $M$. gallinarum was introduced into the cultures 24 to $48 \mathrm{hr}$ before $M$. gallisepticum. The protective effect occurred only with viable $M$. gallinarum organisms and did not involve inhibition of the growth of $M$. gallisepticum. The phenomenon was observed with most strains of $M$. gallisepticum and all strains of $M$. gallinarum tested. A single strain of $M$. hominis failed to protect the ciliary activity of $M$. gallisepticum-infected cultures. Chicken tracheal organ cultures infected with $M$. gallinarum alone showed more prolonged ciliary activity than cultures uninfected with any mycoplasma. The possible reasons for these phenomena are discussed.

\section{REFERENCES}

BLAKE, J. T. 1962. Effects of experimental chronic respiratory disease and infectious bronchitis on pullets. Amer. J. Vet. Res., 23, 847.

BUTLER, M. 1969. Isolation and growth of mycoplasma in human embryo trachea cultures. Nature, Lond., 224, 605.

Cherry, J. D., AND TAYLOR-Robinson, D. 1970a. Large-quantity production of chicken embryo tracheal organ cultures and use in virus and mycoplasma studies. Appl. Microbiol., 19, 658.

CherRy, J. D., AND TAYLOR-Robinson, D. 1970b. Growth and pathogenesis of Mycoplasma mycoides var. capri in chicken embryo tracheal organ cultures. Infect. Immun., 2, 431. 
Cherry, J. D., AND TAYlor-Robinson, D. 1970c. Peroxide production by mycoplasmas in chicken tracheal organ cultures. Nature, Lond., 228, 1099.

CherRY, J. D., AND TAYLOR-RoBINSON, D. 1971. Growth and pathogenicity studies of Mycoplasma gallisepticum in chicken tracheal organ cultures. J. Med. Microbiol., 4, 441.

Collier, A. M., Clyde, W. A., JR, AND Denny, F. W. 1969. Biologic effects of Mycoplasma pneumoniae and other mycoplasmas from man on hamster tracheal organ culture. Proc. Soc. Exp. Biol. Med., 132, 1153.

EDWARD, D. G. ff., AND Freundt, E. A. 1956. The classification and nomenclature of organisms of the pleuropneumonia group. J. Gen. Microbiol., 14, 197.

FABRICANT, J. 1969. Avian mycoplasmas. In The Mycoplasmatales and the L-phase of bacteria, edited by L. Hayflick, New York, p. 621.

HARGREAVES, F. D., AND LeACH, R. H. 1970. The influence of mycoplasma infection on the sensitivity of HeLa cells for growth of viruses. J. Med. Microbiol., 3, 259.

MACPHERSON, I. 1966. Mycoplasmas in tissue culture. J. Cell Sci., 1, 145.

MANCHEE, R. J., AND TAYLOR-RoBINSON, D. 1968. Haemadsorption and haemagglutination by mycoplasmas. J. Gen. Microbiol., 50, 465.

MANCheE, R. J., AND TAYLOR-Robinson, D. 1970. Lysis and protection of erythrocytes by T-mycoplasmas. J. Med. Microbiol., 3, 539.

ReED, Sylvia E. 1971. The interaction of mycoplasmas and influenza viruses in tracheal organ cultures. J. Infect. Dis., 124, 18.

Roberts, D. H. 1964. Serotypes of avian mycoplasma. J. Comp. Path., 74, 447.

TAYLOR-RoBINSON, D., AND BERRY, D. M. 1969. The evaluation of the metabolic-inhibition technique for the study of Mycoplasma gallisepticum. J. Gen. Microbiol., 55, 127.

Thомаs, L. 1970. The toxic properties of $M$. neurolyticum and $M$. gallisepticum. In The role of mycoplasmas and $L$ forms of bacteria in disease, edited by J. T. Sharp, Thomas, Illinois, p. 104. 